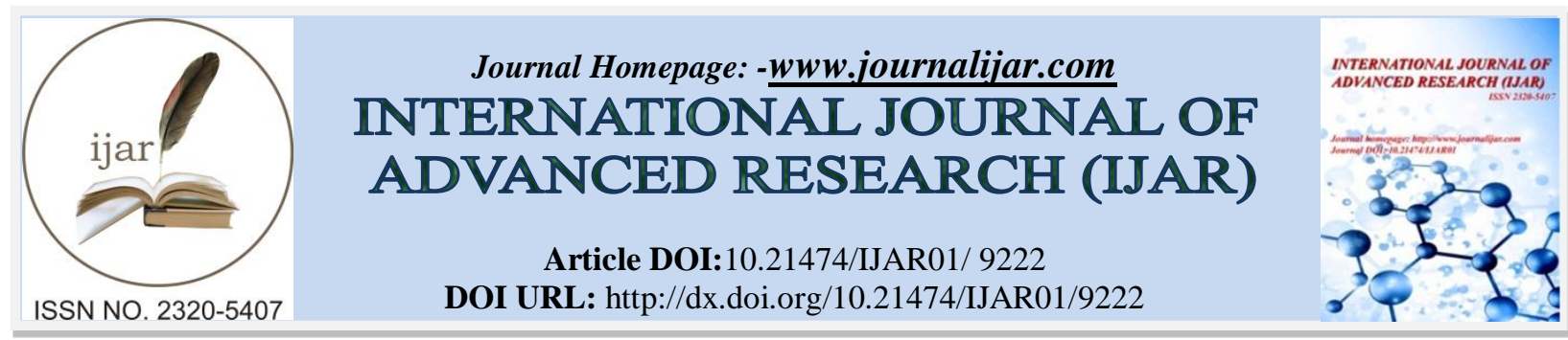

RESEARCH ARTICLE

\title{
CORRELATION BETWEEN PHYSICAL ACTIVITY AND ANTHROPOMETRIC MEASURES IN YOUNG ADULTS.
}

Dr. Twinkle Karia ${ }^{1}$ and Dr. Sukhada Prabhu ${ }^{2}$.

1. B.P.Th, D.Y. Patil University, School Of Physiotherapy, Nerul, Navi Mumbai, Maharashtra.

2. M.P.Th in Cardio Vascular and Respiratory Physiotherapy, Department Of Kinesiotherapeutics, Assistant Professor, D.Y. Patil University, School Of Physiotherapy, Nerul, Navi Mumbai, Maharashtra.

\section{Manuscript Info}

\section{Manuscript History}

Received: 06 April 2019

Final Accepted: 08 May 2019

Published: June 2019

Key words:-

Physical Activity, Anthropometric measures, Obesity, IPAQ, caliper, BMI, Waist circumference, young adults.

\begin{abstract}
Background: Body compositions and sedentary lifestyle play an important role in increasing obesity in an individual. The real reason for this can be ought to be sedentary lifestyle or decreased physical activity in young adults. Thus, this study would help us find correlation between levels of Physical activity (PA) and anthropometric measures in young adults and help us create awareness about importance of Physical activity and reduce risk factors and promote healthy lifestyle. Aim: To assess physical activity and anthropometric measures in young adults. Objectives: 1) To assess Physical activity using IPAQ in young adults. 2) To assess BMI, waist circumference and body fat percentage with skinfold caliper using Jackson Pollock calculator in young adults. 3) To correlate physical activity and anthropometric measures. Methodology: A total of 80 young adults of the age group 17 to 22 years of age were taken for this study. The design of this study is Observational study. Their physical activity was calculated using International Physical Activity Questionnaire (IPAQ), body mass index (BMI) and waist circumference were also calculated. Body fat percentage for 3 site skin fold thickness using skinfold caliper was calculated using Jackson Pollock calculator. Results: 1) Direct correlation exists between PA and BMI, PA and Age and PA and Waist circumference in both males and females. 2) Inverse correlation exists between PA and body fat percentage in males and direct correlation in females. 3) Inverse correlation exists between PA and Body Density in females and direct correlation in males.
\end{abstract}

Copy Right, IJAR, 2019,. All rights reserved.

\section{Introduction:-}

Any bodily movements that require energy expenditure produced by skeletal muscles, is known as Physical Activity (PA). ${ }^{[1]}$ Physical activity is generally classified as Low, Moderate and High levels of Physical activity and is measured in METs as follows:

1. Low levels of Physical activity- Less than 3 METs

2. Medium level of Physical Activity- 3 to 6 METs

3. High Level of Physical Activity- More than 6 METs 
Physical activity can be assessed using direct and indirect ways. Direct method includes pedometers, accelometers whereas indirect assessment of Physical activity includes various scales such as International Physical activity questionnaire (IPAQ), Global Physical Activity Questionnaire (GPAQ), Physical Activity readiness (PAR), Duke's, etc. IPAQ is a self-administered questionnaire which has acceptable validity when assessing levels and patterns of physical activity in healthy adults. IPAQ is used to measure physical activity in adults ranging from 15 to 69 years of age. There are two forms of IPAQ - long form and short form. There are 7 questions in short form and 27 questions in long form. Physical inactivity results in abnormalities of various body compositions.

Anthropometric measures involve systemic body composition measurements. There are various methods of assessing body compositions such as calculation of BMI, waist circumference, Skinfold thickness, mid arm circumference, waist to hip ratio, hydrostatic weighing, etc. Body mass index roughly measures your body fat. It is an easy and zero cost self-administered method and hence it is widely used. Waist circumference (WC) measures central obesity. For measuring abdominal obesity, WC is the best indicator as compared with waist to hip ratio. ${ }^{[2]}$ Skinfold measurements are utilized to assess body composition. Once caliper is bought, test costs are minimal. A study carried out by Gregory L. Stalker in 2011 approved by UNLV Biomedical institutional review board suggests that the results of the study has demonstrated the Body Caliper to be a reliable and valid instrument and method for evaluating body composition. ${ }^{[3]}$ In order to measure body fat percentage using skinfold caliper, Jackson Pollock calculator can be used. It calculates body fat percentage using formula that is separate for male and female. There are various methods namely- 3 site method, 7 site method, etc. Estimation of body fat percentage using the Pollock 3 -site method has comparable accuracy. ${ }^{[4]}$

Body compositions can be categorized as underweight, normal or obese. But renowned obesity is noticed in younger adults due to physical inactivity and sedentary lifestyle. Accumulation of excessive or abnormal fat that presents a risk to health is defined as Obesity. ${ }^{[5]}$ As a result of sedentary lifestyle, physical activity is decreasing drastically among younger adults. Over the past decades, burden of obesity has increased rapidly. ${ }^{[6]}$ In many parts of the world, it is endemic. Obesity is most neglected though blatantly visible public health problems. ${ }^{[7]}$ A study carried out by Dr. pinnamaneni Siddhartha, India in population $\geq 20$ years of age suggested that prevalence of generalized, abdominal and combined obesity was 56\%, 71.2\% and 51.3\% respectively. ${ }^{[8]}$ Most of the individuals in India are inactive. Physical inactivity and Obesity can result in various complications such as high blood pressure, diabetes, cancer, depression, etc. Hence, it's high time we focus on these risks and assess correlation between physical activity and various anthropometric measures.

Thus, this study would help us find levels of physical activity and anthropometric measures like Body mass index (BMI), waist circumference and body fat percentage in younger adults and it would help us assess correlation between them. WHO suggests that Regular and adequate levels of physical activity in adults would:

1. reduce the risk of the risk of falls hypertension, diabetes, coronary heart disease, stroke;

2. improve functional health and bone density; and

3. Improve fundamental to energy balance and weight control, acting as key determinant of energy expenditure.

Hence, finding relation between these components would help us find risk factors and create awareness about being physically active and have a healthy lifestyle.

\section{Aim:}

To assess correlation between physical activity and anthropometric measures in young adults.

\section{Objectives:}

1. To assess Physical activity using IPAQ in young adults

2. To assess BMI and waist circumference in young adults.

3. To assess body fat percentage with skinfold caliper using Jackson Pollock calculator in young adults.

4. To correlate physical activity and anthropometric measures.

\section{Material and Methodology:-}

A written consent was be taken by the individual participating in the study. A proforma including demographic details such as name, age, gender, weight, height, etc. was prepared. An ethical clearance was obtained from the 
institution. The study was conducted on 80 subjects between 17 to 22 years of age. The subjects belonged to D.Y. Patil School of Physiotherapy, and various societies of Kandivali.

\section{Inclusion criteria:-}

Younger adults from 17 to 22 years of age

\section{Exclusion criteria:-}

\section{Adults with any underlying}

1. Respiratory disorders like

2. Bronchial Asthma

3. Chronic Obstructive Pulmonary Disease (COPD);

4. Cardiovascular disorders like

5. congestive heart failure,

6. Myocardial infarction or

7. Neurological problems etc.

\section{Unwilling to participate.}

\section{Tools \& Materials:-}

1. International physical activity questionnaire (IPAQ)

2. Measuring tape

3. Weighing scale

4. Skinfold caliper

5. Jackson Pollock calculator

\section{Plan of Study:-}

\section{Assessment of Physical Activity}

In order to measure the level of physical activity, a standard and validated questionnaire called international physical activity questionnaire (IPAQ) was used. The IPAQ consists of 5 domains and has 27 questions. The domains of the questionnaire are as follows:

1. Part 1 -job related physical activity

2. Part 2- transportation physical activity

3. Part 3- housework, house maintenance and caring for family

4. Part 4- recreation, sport and leisure- the physical activity

5. Part 5- Time spent sitting

Scoring of IPAQ was done following IPAQ standard scoring protocol and were classified as Low, Moderate or High levels of Physical Activity.

\section{Anthropometric measures}

BMI- height was measured using measuring tape in $\mathrm{cms}$ and weight using calibrated weighing scale in $\mathrm{kgs}$. $\mathrm{BMI}=$ $\mathrm{M} /(\mathrm{H} \times \mathrm{H})$, where $\mathrm{M}=$ body mass in kilograms and $\mathrm{H}=$ height in meters.

Waist circumference - was measured at the midpoint between the lower margin of the least palpable rib and the top of the iliac crest. ${ }^{[9]}$ The subjects stood with feet close together, arms at the side and body weight evenly distributed. The subject was relaxed, and the measurements were taken at the end of a normal expiration. Each measurement was repeated twice; the average was calculated. If the difference between the two measurements exceeded $1 \mathrm{~cm}$, the two measurements were repeated. ${ }^{[9]}$

Skinfold caliper was used to measure the body fat percentage. A study carried out by Gregory L. Stalker suggests that the results of the study has demonstrated the Body Caliper to be a reliable and valid instrument and method for evaluating body composition. ${ }^{[10]} 3$ site method was used. It was done on the right side of the body following protocol at 3 sites, namely: 


\section{For Males:}

1. Chest

2. Abdomen

3. Thigh

\section{For Females:}

1. Triceps

2. Suprailiac

3. Thigh

\section{Procedure:-}

Subject's skin was firmly grasped between thumb and index finger and lifted up. The skinfold included two thicknesses; one of skin and one of the subcutaneous fat, but no muscle or fascia. Caliper was placed at a 90 degree angle to the skinfold approximately $1 \mathrm{~cm}$ below the fingers. Slightly the pressure between the fingers was released, holding the skinfold, greater pressure was applied by the caliper. Releasing the pressure, reading was taken. Three readings were taken and average was used for the further study.

\section{Statistical data presentation and analysis}

All the statistical analysis was done using SPSS version 22.0 for windows. Pearson correlation test was done. A p value of $<0.05$ was considered statistically significant.

Table 1:-

\begin{tabular}{|l|l|l|}
\hline & Mean \pm SD Males & Mean \pm SD Females \\
\hline Body mass index & $24.96 \pm 6.38$ & $22.43 \pm 3.76$ \\
\hline Waist circumference & $83.70 \pm 13.16$ & $72.91 \pm 10.45$ \\
\hline Body density & $1.05 \pm 0.02$ & $1.04 \pm 0.01$ \\
\hline Body fat percentage & $20.91 \pm 7.12$ & $27.62 \pm 4.52$ \\
\hline
\end{tabular}

Graph 1:-Distribution according to age

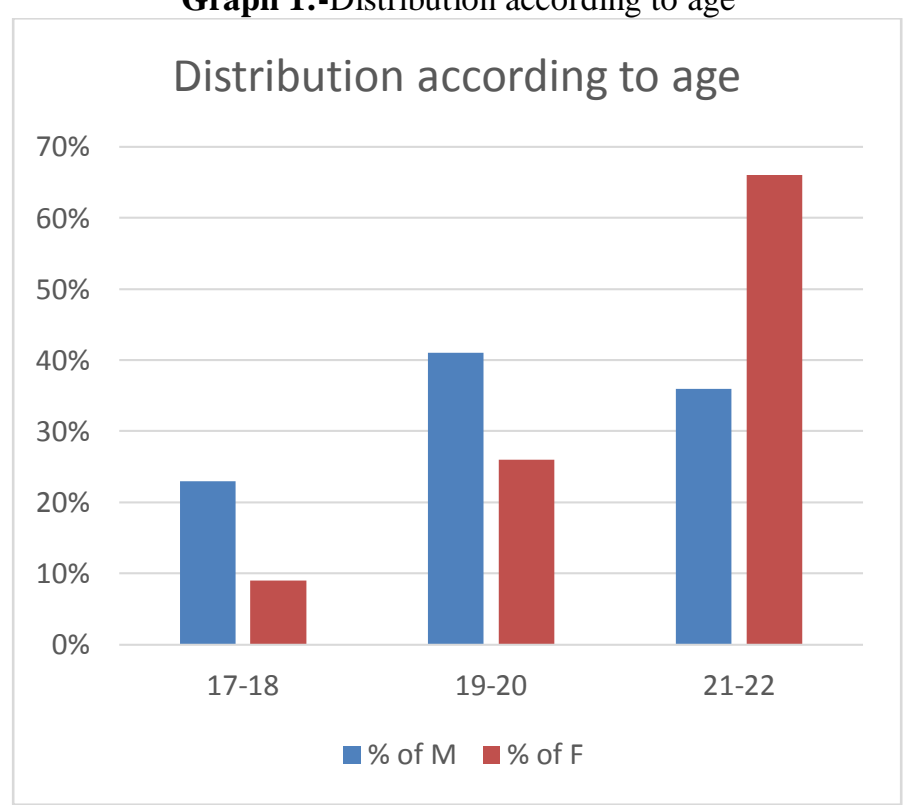

Table 2:-Distribution according to age

\begin{tabular}{|l|l|l|l|l|}
\hline Age Intervals & Male & percentage of Males & Females & Percentage of Females \\
\hline $17-18$ & 5 & $23 \%$ & 5 & $9 \%$ \\
\hline $19-20$ & 9 & $41 \%$ & 15 & $26 \%$ \\
\hline $21-22$ & 8 & $36 \%$ & 38 & $66 \%$ \\
\hline
\end{tabular}




\section{Inference:}

As per the graph, $23 \%$ of males fall in class interval of $17-18$ years of age, $41 \%$ fall in age group of $19-20$ years and $36 \%$ are in class interval of 21-22 years of age. Also, 9\% of females are included in class interval of 17-18 years of age, $26 \%$ are in class interval of 19-20 years of age and 66\% are in class interval of 21-22 years of age.

Graph 2: Distribution according to gender

\section{Distribution according to gender}

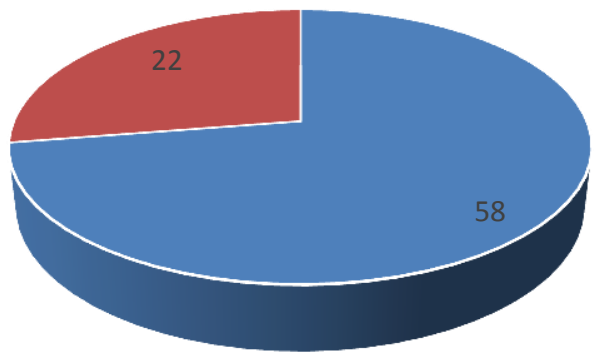

Table 3:-Distribution according to gender

\begin{tabular}{|l|l|l|}
\hline Gender & No. Of & Percentage \\
\hline Females & 58 & $73 \%$ \\
\hline Males & 22 & $28 \%$ \\
\hline
\end{tabular}

Inference:

As per the pie chart, out of the total subjects, $73 \%$ subjects were females and $28 \%$ of subjects were male.

Graph 3: Distribution according to Body Mass Index

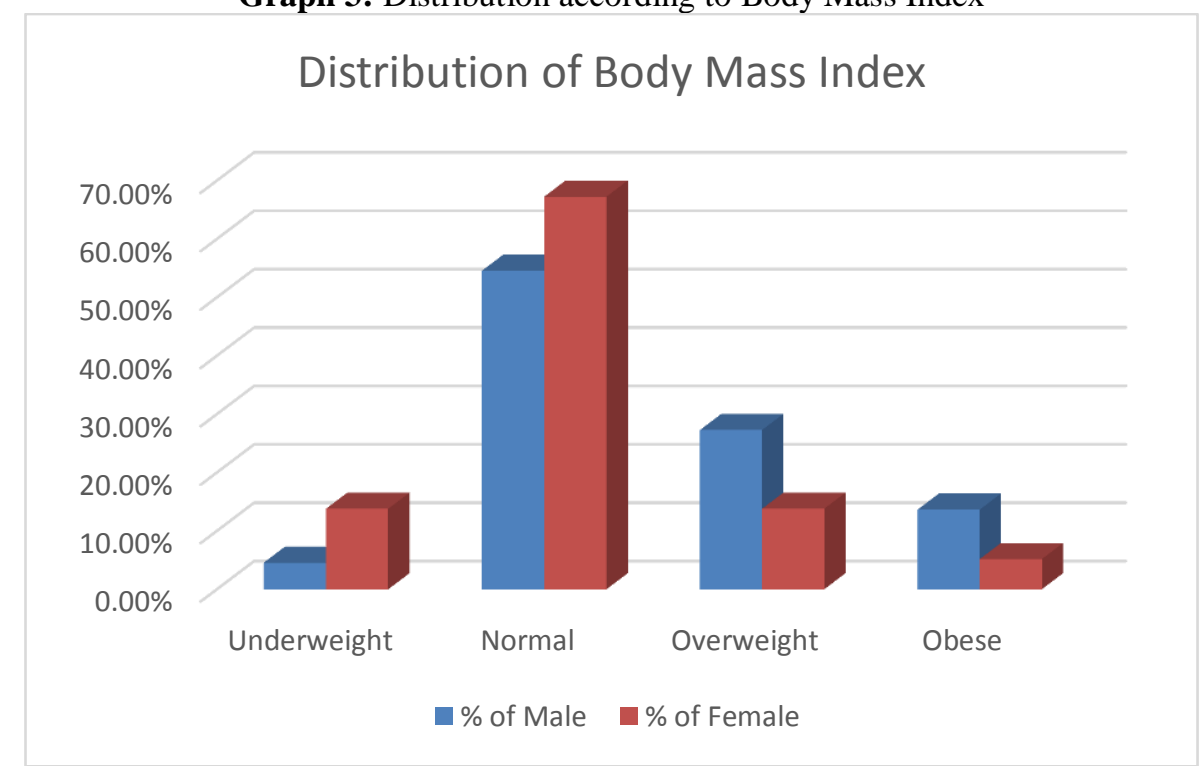

Table 4:-Distribution according to Body Mass Index

\begin{tabular}{|l|l|l|l|l|}
\hline BMI & Male & Female & \% of Male & $\%$ of Female \\
\hline Underweight & 1 & 8 & $4.55 \%$ & $13.79 \%$ \\
\hline
\end{tabular}




\begin{tabular}{|l|l|l|l|l|}
\hline Normal & 12 & 39 & $54.55 \%$ & $67.24 \%$ \\
\hline Overweight & 6 & 8 & $27.27 \%$ & $13.79 \%$ \\
\hline Obese & 3 & 3 & $13.64 \%$ & $5.17 \%$ \\
\hline Total & 22 & 58 & $100 \%$ & $100 \%$ \\
\hline
\end{tabular}

Inference:

As per the graph, $4.55 \%$ of males and $13.79 \%$ of females are underweight, $54.55 \%$ males a67.24\% of females have Normal Body mass index, $27.27 \%$ of males and $13.79 \%$ of females are overweight and $13.64 \%$ of males and $5.17 \%$ of females are obese.

Graph 4:-Distribution according to Waist circumference

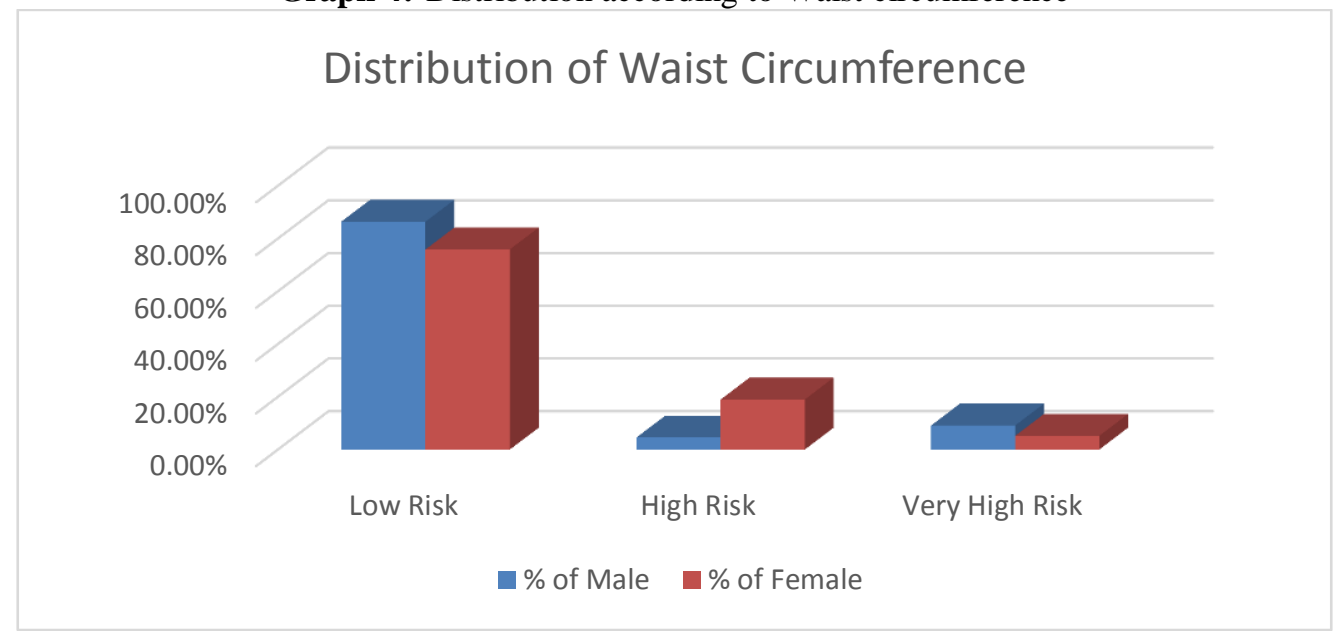

Table 5:-Distribution according to Waist circumference

\begin{tabular}{|l|l|l|l|l|}
\hline Waist Circumference & Male & Female & $\%$ of Male & \% of Female \\
\hline Low Risk & 19 & 44 & $86.36 \%$ & $75.86 \%$ \\
\hline High Risk & 1 & 11 & $4.55 \%$ & $18.97 \%$ \\
\hline Very High Risk & 2 & 3 & $9.09 \%$ & $5.17 \%$ \\
\hline Total & 22 & 58 & $100.00 \%$ & $100.00 \%$ \\
\hline
\end{tabular}

\section{Inference:}

As per the graph, according to waist circumference of subjects, $86.36 \%$ of males and $75.86 \%$ of females are at low risk of getting cardiovascular diseases, $4.55 \%$ of males and $18.97 \%$ of females are at high risk of getting cardiovascular diseases and $9.09 \%$ of males and $5.17 \%$ of females have very high risk of getting cardiovascular diseases. 
Graph 5:-Distribution according to Body Fat

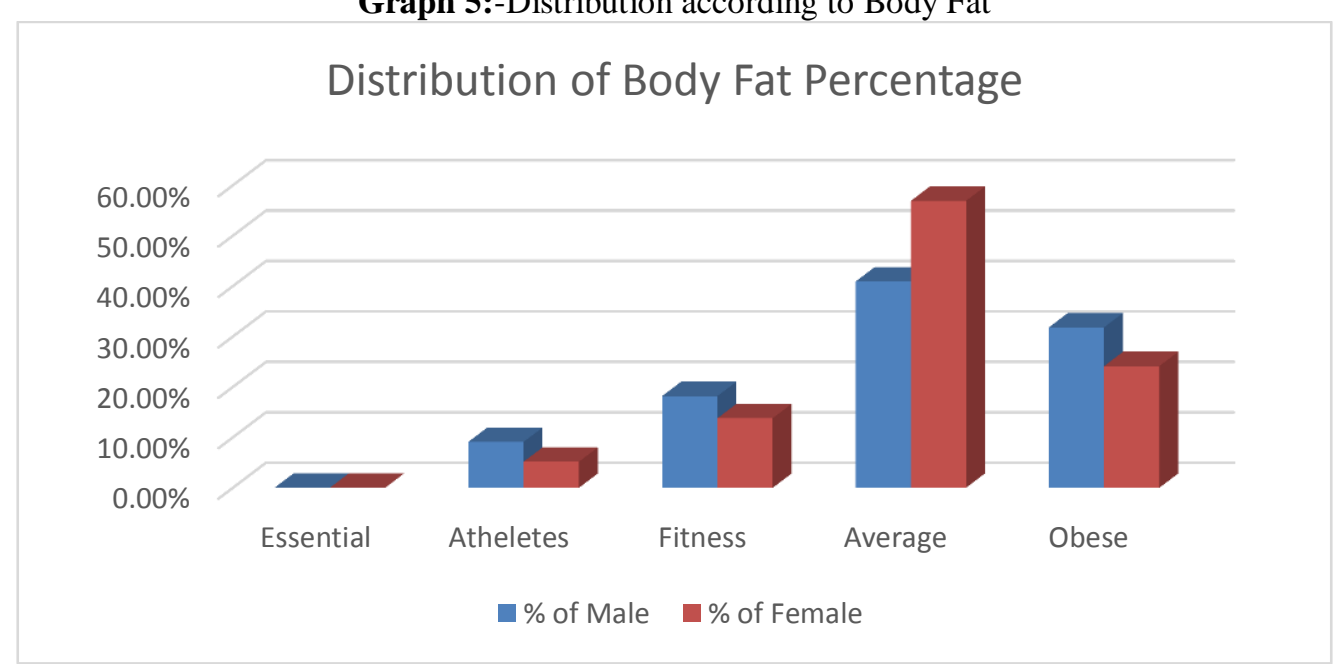

Table 6:-Distribution according to Body Fat

\begin{tabular}{|l|l|l|l|l|}
\hline Body Fat \% & Male & Female & \% of Male & \% of Female \\
\hline Essential & 0 & 0 & $0.00 \%$ & $0.00 \%$ \\
\hline Athletes & 2 & 3 & $9.09 \%$ & $5.17 \%$ \\
\hline Fitness & 4 & 8 & $18.18 \%$ & $13.79 \%$ \\
\hline Average & 9 & 33 & $40.91 \%$ & $56.90 \%$ \\
\hline Obese & 7 & 14 & $31.82 \%$ & $24.14 \%$ \\
\hline Total & 22 & 58 & $100.00 \%$ & $100.00 \%$ \\
\hline
\end{tabular}

Inference: As per graph, $9.09 \%$ of males and $5.17 \%$ of females fall in athletes' category, $18.18 \%$ of males and $13.79 \%$ of females are fit, $40.91 \%$ of males and $56.90 \%$ of females have average body fat percentage and $31.82 \%$ of males and $24.14 \%$ of females are obese.

Graph 6:-Distribution according to Physical Activity

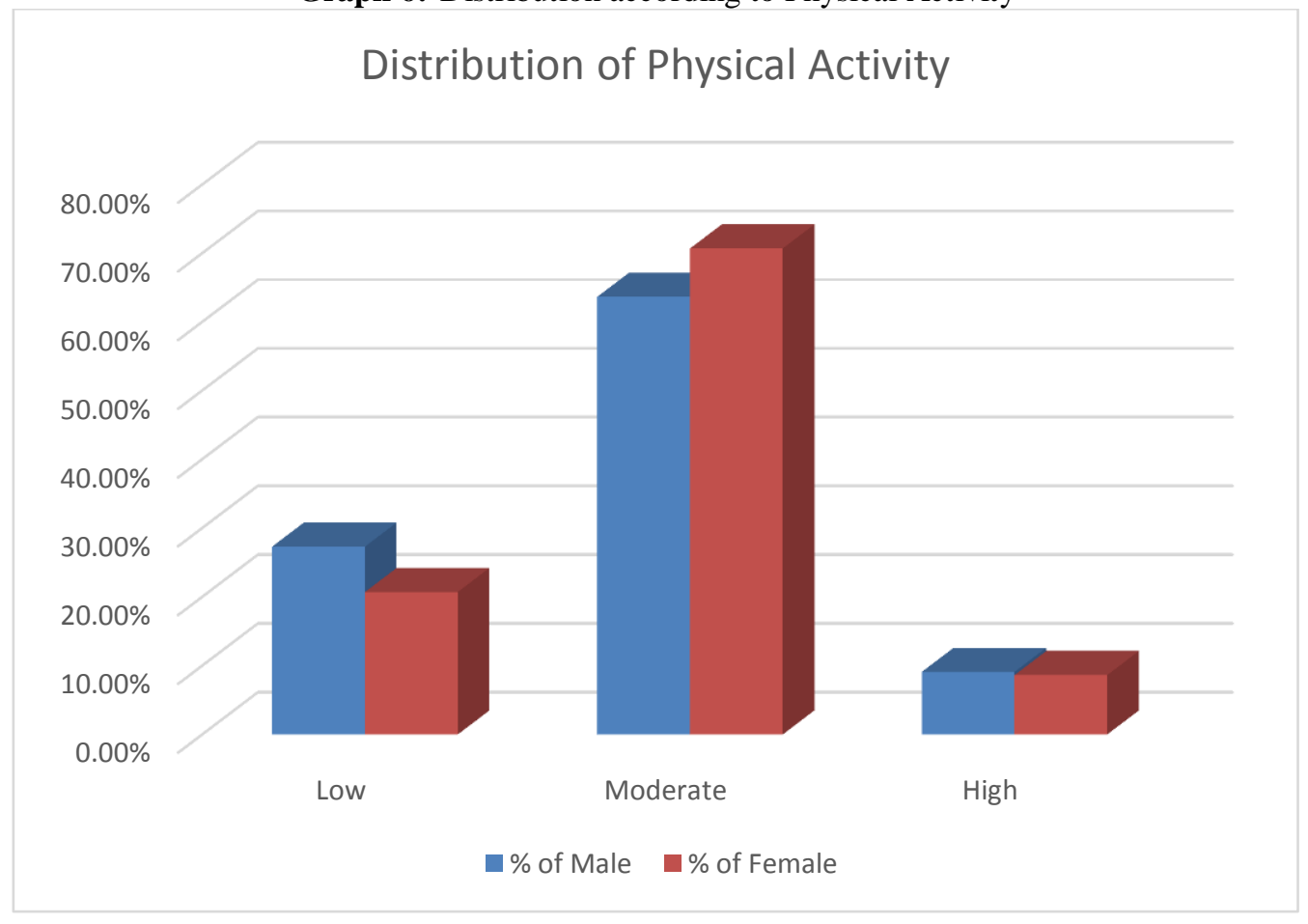


Table 7:-Distribution according to Physical Activity

\begin{tabular}{|l|l|l|l|l|}
\hline Physical Activity & Male & Female & $\%$ of Male & $\%$ of Female \\
\hline Low & 6 & 12 & $27.27 \%$ & $20.69 \%$ \\
\hline Moderate & 14 & 41 & $63.64 \%$ & $70.69 \%$ \\
\hline High & 2 & 5 & $9.09 \%$ & $8.62 \%$ \\
\hline Total & 22 & 58 & $100.00 \%$ & $100.00 \%$ \\
\hline
\end{tabular}

\section{Inference:}

As per graph, 27.27 of males and $20.69 \%$ of females have low levels of physical activity, 63.64\% of males and $70.69 \%$ of females have moderate levels of physical activity and $9.09 \%$ of males and $8.62 \%$ of females have high levels of physical activity.

Graph 7:-Correlation between physical activity and body mass Index (BMI)
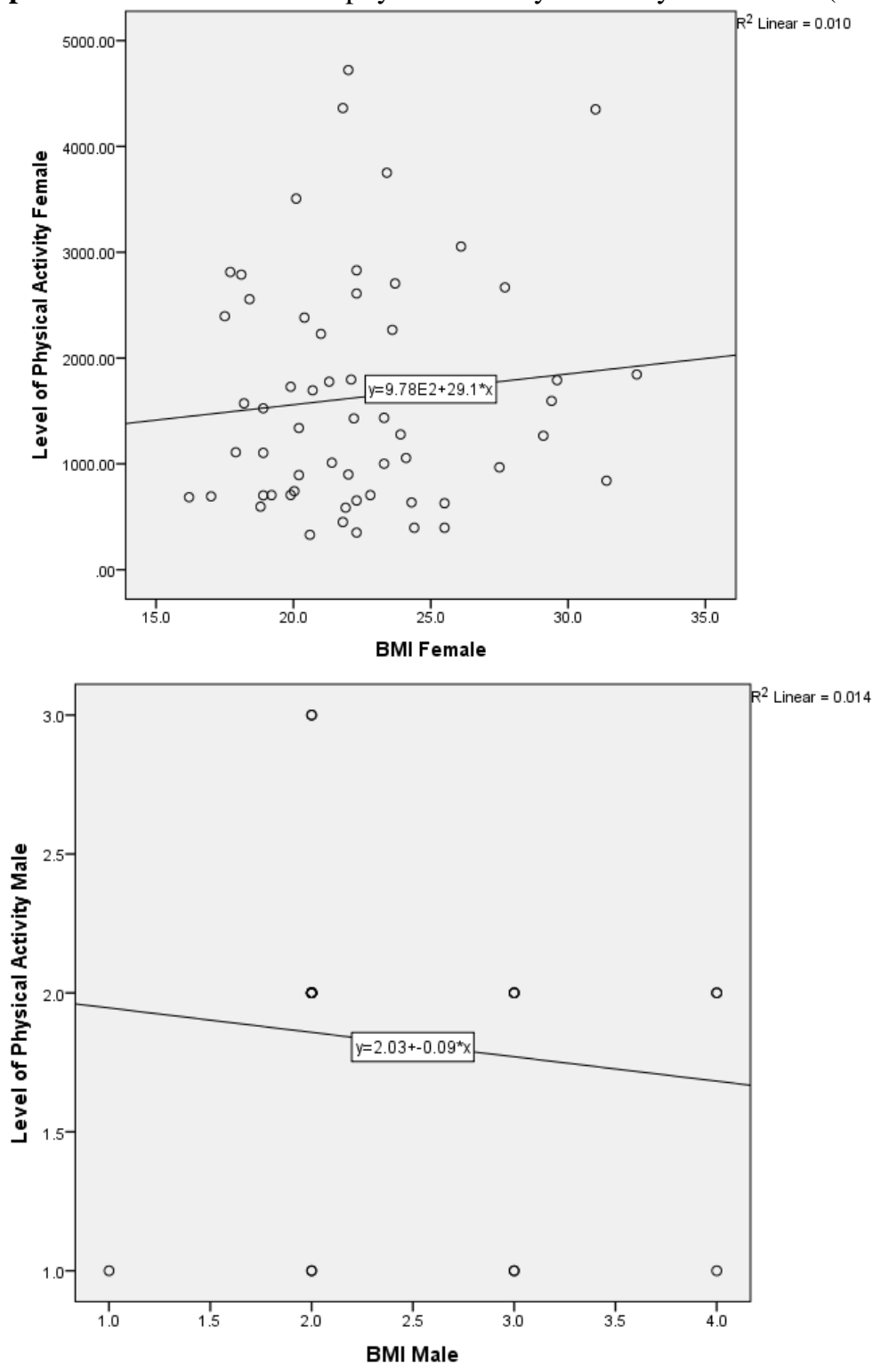

Table 8:-Correlation between physical activity and body mass Index (BMI)

\begin{tabular}{|l|l|l|}
\hline & Male & Female \\
\hline P value & 0.824 & 0.454 \\
\hline
\end{tabular}




\begin{tabular}{|l|l|l|}
\hline Pearson correlation & 0.050 & 0.100 \\
\hline
\end{tabular}

\section{Inference:}

As per the graph, correlation between Physical activity and Body mass index (BMI) for both males and females is non-significant but there exists a direct correlation between them.
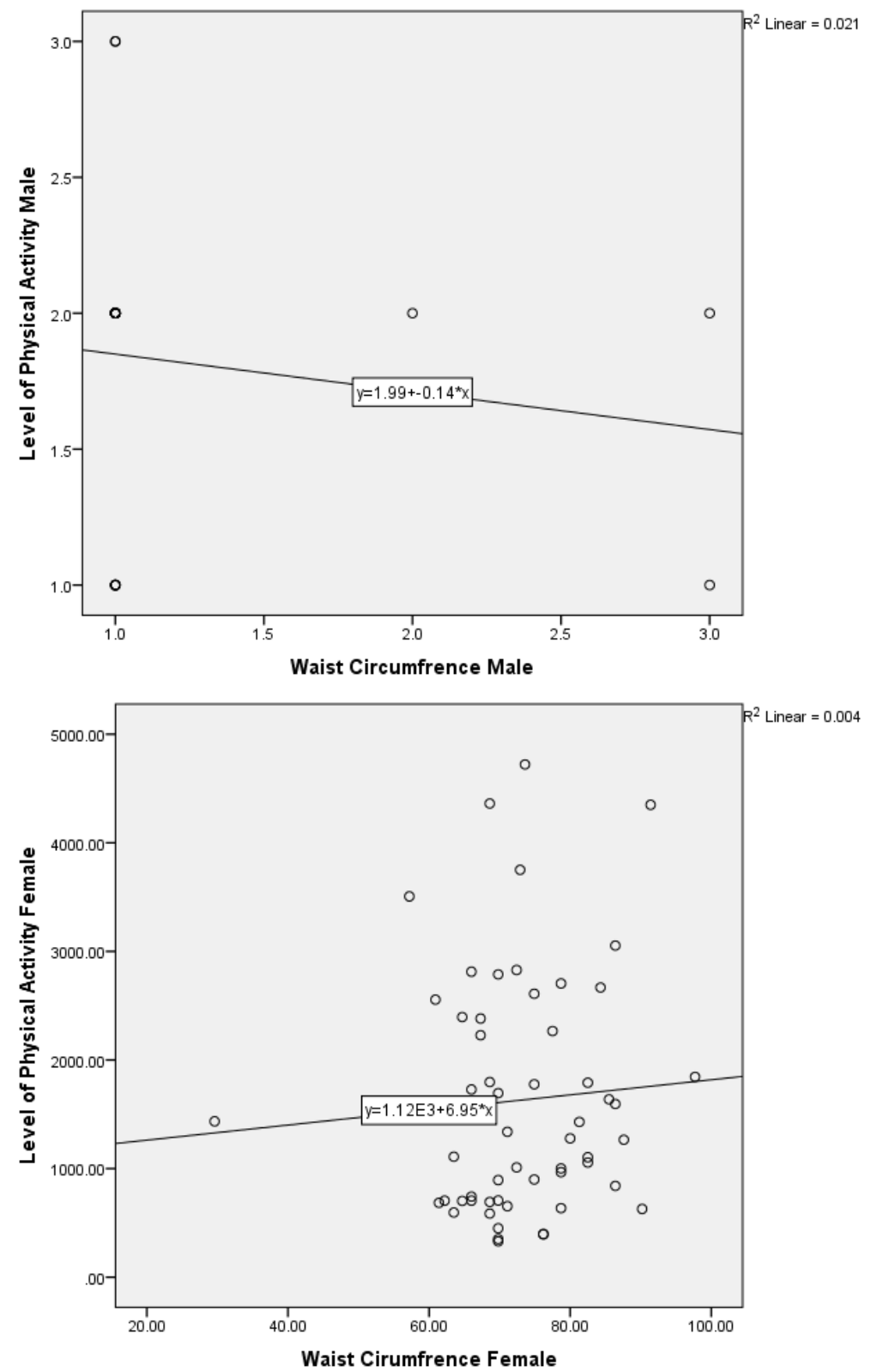

Graph 8:-Correlation between Physical Activity and Waist Circumference

Table 9:-Correlation between Physical Activity and Waist Circumference

\begin{tabular}{|l|l|l|}
\hline & Male & Female \\
\hline P value & 0.861 & 0.620 \\
\hline Pearson correlation & 0.040 & 0.067 \\
\hline
\end{tabular}




\section{Inference:}

As per the graph, correlation between Physical activity and waist circumference for both males and females is nonsignificant but there exists a direct correlation between them.

Graph 9: Correlation between Physical Activity and Body fat percentage
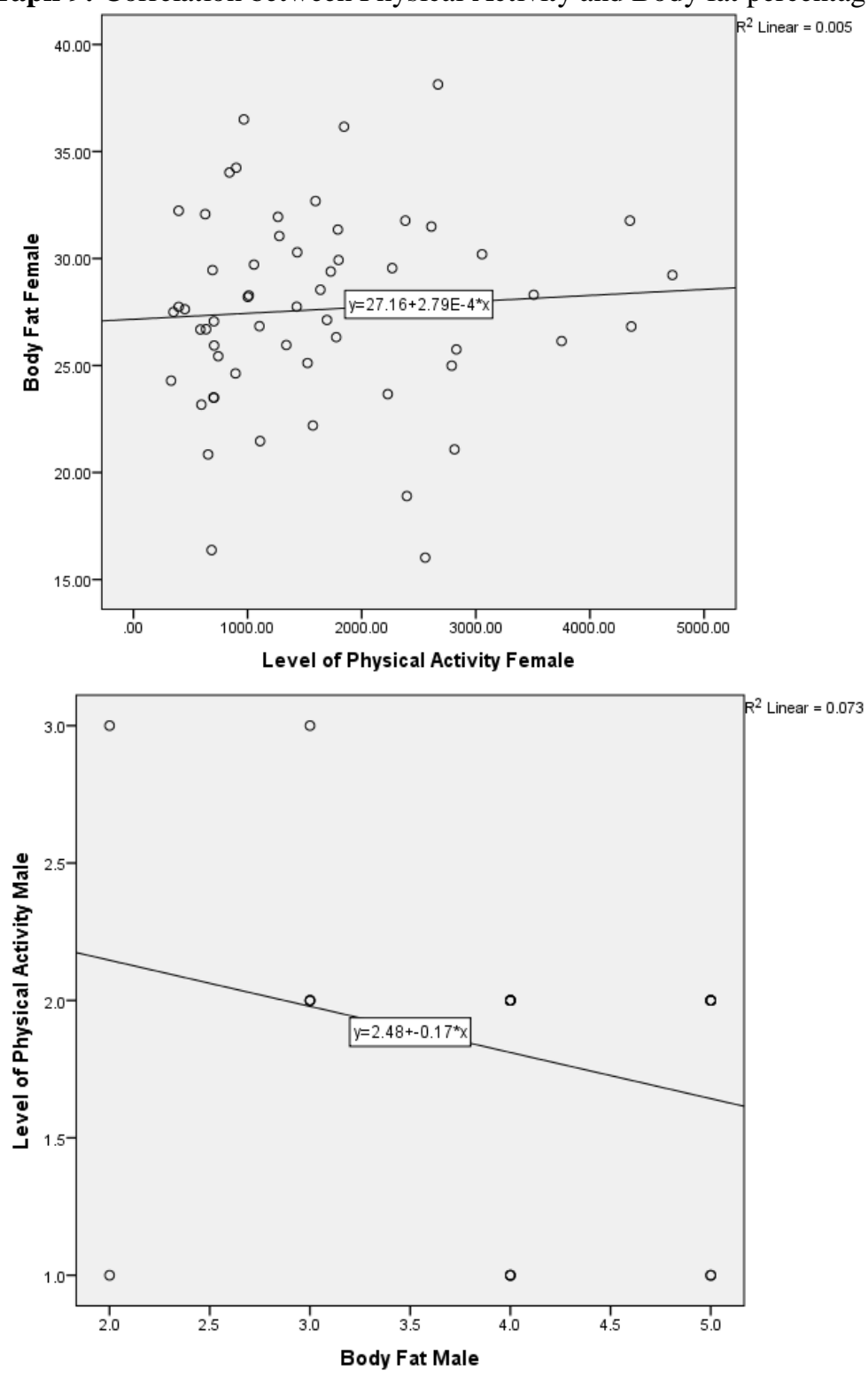

Table 10:-Correlation between Physical Activity and Body fat percentage

\begin{tabular}{|l|l|l|}
\hline & Male & Female \\
\hline P value & 0.739 & 0.615 \\
\hline Pearson correlation & 0.075 & 0.67 \\
\hline
\end{tabular}

\section{Inference:}

As per the graph, correlation between Physical activity and Body fat percentage for both males and females is nonsignificant but there exists an inverse correlation between them in males and direct correlation between them in females. 
Graph 10:-Correlation between Physical Activity and Body Density
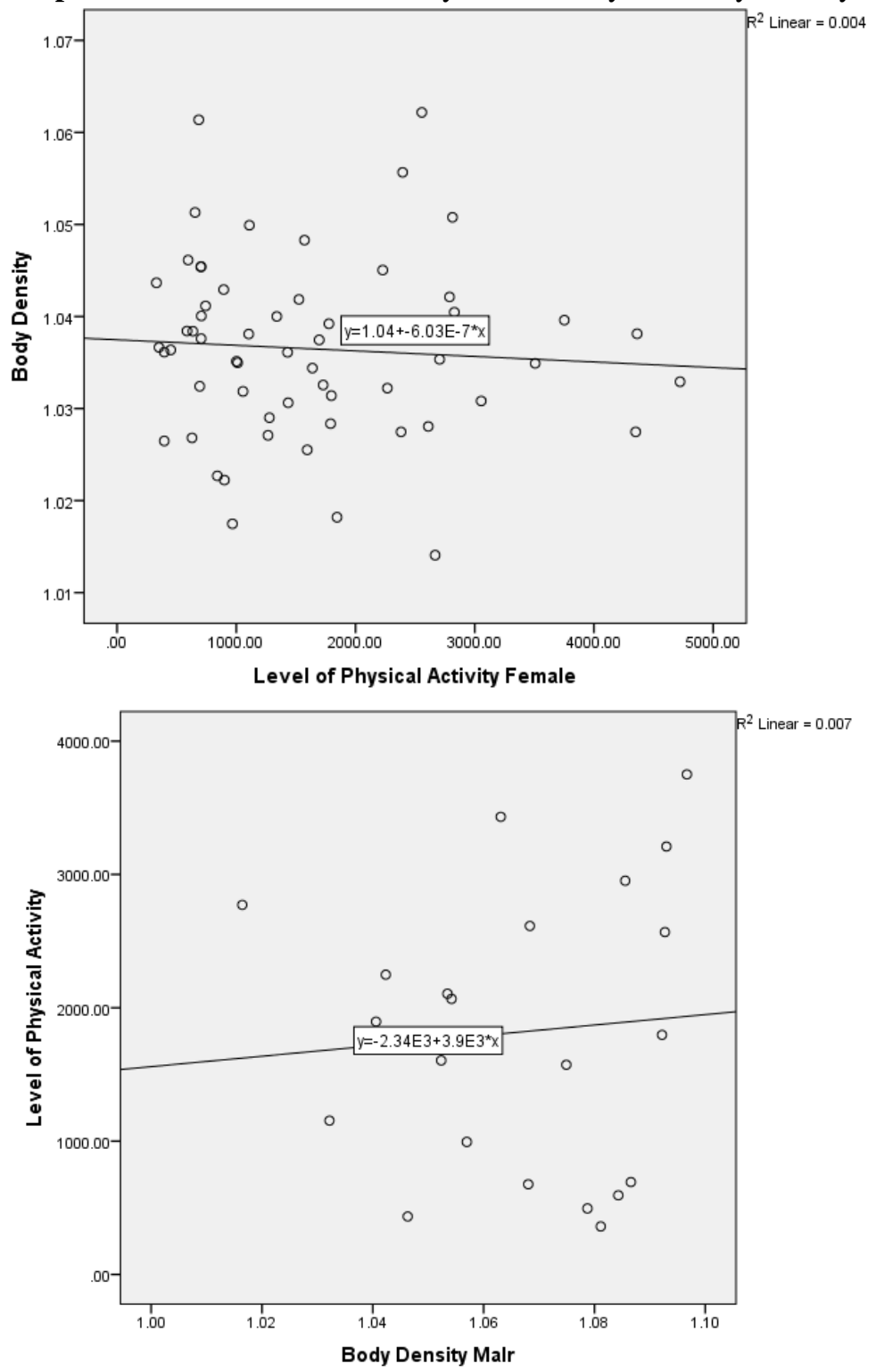

Table 11:-Correlation between Physical Activity and Body Density

\begin{tabular}{|l|l|l|}
\hline & Male & Female \\
\hline P value & 0.713 & 0.618 \\
\hline Pearson correlation & 0.083 & 0.067 \\
\hline
\end{tabular}

Inference:

As per the graph, correlation between Physical activity and Body Density for both males and females is nonsignificant but there exists a direct correlation between them in males and inverse correlation between them in females. 

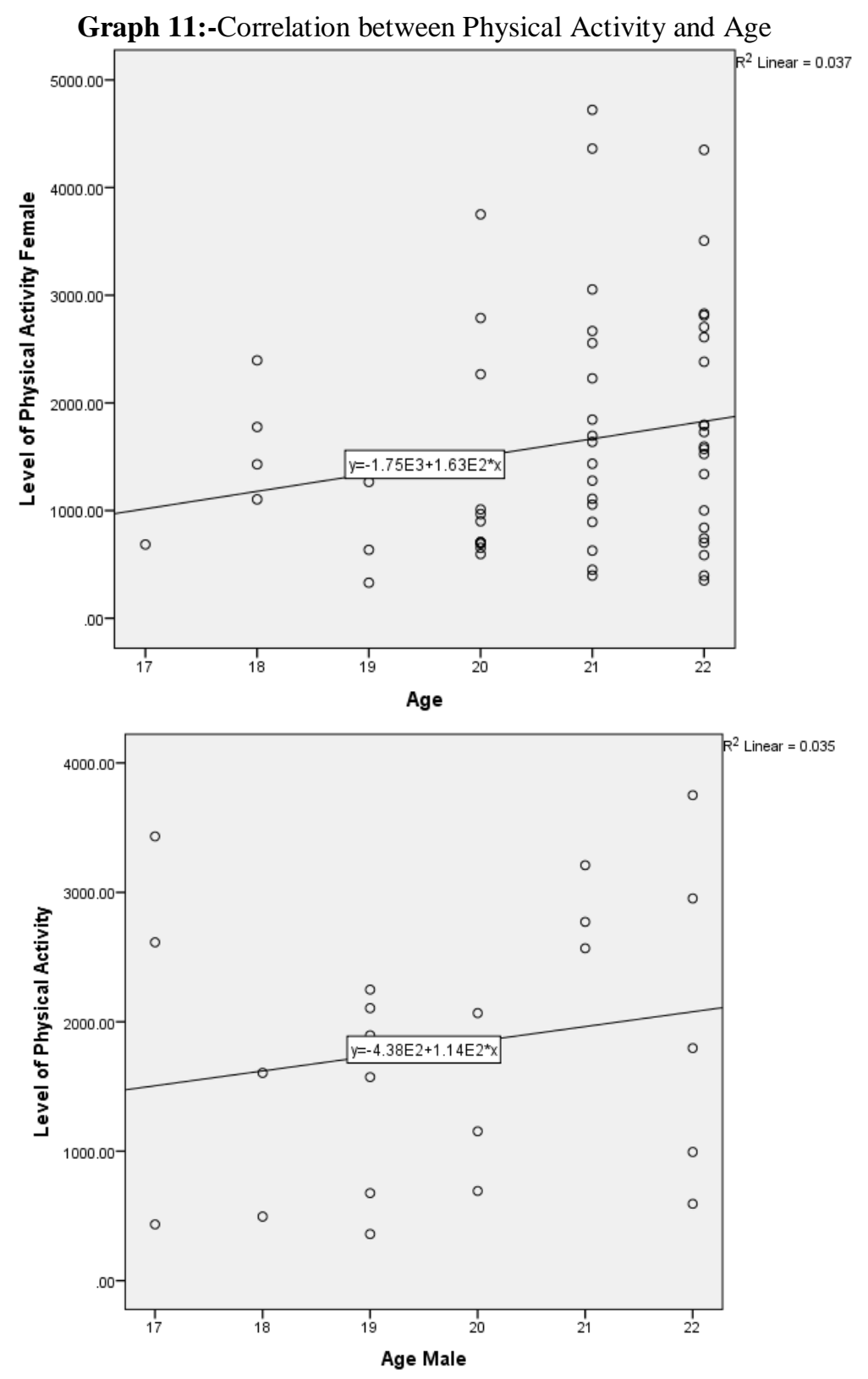

Table 12:-Correlation between Physical Activity and Age

\begin{tabular}{|l|l|l|}
\hline & Male & Female \\
\hline P value & 0.402 & 0.150 \\
\hline Pearson correlation & 0.188 & 0.191 \\
\hline
\end{tabular}

\section{Inference:}

As per the graph, correlation between Physical activity and Age for both males and females is non-significant but there exists a direct correlation between them.

\section{Discussion:-}

Assessment of levels of Physical activity and Anthropometric measures was conducted in present research study amongst 80 young adults belonging to age group of 17 to 22 years of age. It was an observational study carried out to assess correlation between Physical Activity and Anthropometric measures in young adults. The subjects were taken from D.Y. Patil University School of Physiotherapy and various societies of Kandivali, Mumbai. 
As per graph 1, Out of 80 subjects, $73 \%$ of the subjects were females and $27 \%$ were males. Graph number 2 shows that $23 \%$ of males and $9 \%$ of females are included in class interval of $17-18$ years of age. $41 \%$ of males and $26 \%$ of females are included in 19-20 years of age. 36\% of males and 66\% of females are included in 21-22 years of age. This suggests that maximum percentage of subjects in males are included in class interval of 19-20 years of age and maximum percentage of subjects in females are included in class interval of 21-22 years of age.

Graph number 3 shows distribution according to Body Mass Index (BMI), maximum number of subjects: males (4.55\%) and females (13.79\%) have normal BMI, followed by $27.27 \%$ of males and $13.79 \%$ of females included in overweight category, followed by $13.64 \%$ of males being obese and $4.55 \%$ of males being underweight and $13.79 \%$ of females being underweight and $5.17 \%$ of females being obese. The variation in BMI among young adults could be because of physical inactivity, unhealthy lifestyle or any underlying disease. High percentage of population belonging to overweight category could be because the population belonging to this age group have prolonged periods of sitting due to lectures, studies, addiction to technologies such as video games, mobile phones, etc. which leads to lack of physical activity.

According to waist circumference distribution, graph 4 shows that maximum percentage of males $(86.36 \%)$ and females $(75.86 \%$ ) have low risk of getting cardiovascular disorders. $4.55 \%$ of males and $18.97 \%$ of females have high risk of cardiovascular disorders and $9.09 \%$ of males and $5.17 \%$ of females have very high risk of cardiovascular disorders. The variation in waist circumference in young adults could be because of high BMI, physical inactivity, unhealthy or sedentary lifestyle. Poor nutrition and improper dietary habits like excessive consumption of fried food, junk food, aerated drinks, etc. which have been adapted from western culture attributes to obesity.

According to body fat percentage, distribution, graph 5 shows that maximum percentage of subjects: male (40.91\%) and females $(56.90 \%)$ are considered to have average body fat percentage, followed by $31.82 \%$ of males and $24.24 \%$ of females concluded to be obese. $9.09 \%$ of males and $5.17 \%$ of females fall under athletes category, $18.18 \%$ of males and $13.79 \%$ of females are observed to have fat percentage that concludes them to be fit. High percentage of population belonging to obese category could be attributed to genes, sedentary lifestyle, poor eating habits like consumption of high carbohydrates and high fat food, lack of exercise, etc.

Graph number 6 shows the distribution according to levels of Physical Activity, which suggests that $63.64 \%$ of males and $70.69 \%$ of females are observed to have moderate levels of Physical Activity, followed by $27.27 \%$ of males and $20.69 \%$ of females are observed to have low levels of Physical Activity and $9.09 \%$ of males and $8.62 \%$ of females are observed to High levels of Physical Activities. The majority subjects had moderate or low levels of physical activity; this could be because of sedentary lifestyle, unhealthy eating habits, unaware about importance of being physically active, risk factors associated with decreased levels of physical activity, etc.

Correlation between Physical Activity and Various Anthropometric Measures were observed. Graph number 7 shows that Correlation between Physical activity and Body mass index (BMI) for both males and females is nonsignificant ( $\mathrm{p}=0.824$ in males and $\mathrm{p}=0.454$ in females) but there exists a direct correlation (Pearson correlation= 0.050 in males and 0.100 in females) between them which means that as Physical activity increases, body mass index also increases. The correlation between them could be non- significant as majority of subjects taken for this study have normal BMI. Leila Jaafari suggests that all Physical fitness factors (PFF) like aerobic fitness, abdominal Muscles endurance, and feet muscles explosive strength, except flexibility, are in inverse correlation with BMI so that any increase in some PFF would lead to some decrease in BMI. ${ }^{[11]}$

Graph number 8 shows that Correlation between Physical activity and waist circumference for both males and females is statistically non-significant ( $\mathrm{p}=0.861$ in males and $\mathrm{p}=0.620$ in females) but there exists a direct correlation (Pearson correlation $=0.040$ in males and Pearson correlation $=0.067$ in females) between them which means that as Physical activity increases, waist circumference increases. This could again be because most of our subjects have normal BMI and thus are at lower risk of getting cardiovascular disorders.

Graph number 9 shows that Correlation between Physical activity and Body fat percentage for both males and females is non-significant ( $\mathrm{p}=0.739$ in males and $\mathrm{p}=0.615$ in females) but there exists an inverse correlation between them in males (Pearson correlation $=0.075$ ) and direct correlation (Pearson correlation $=0.67$ ) between them in females. The correlation in males suggests that as physical activity increases, body fat percentage decreases, this is 
because among male subjects majority males have average body fat percentage and the correlation is non-significant because the number of sample taken for the study are less and maximum have normal BMI. The correlation between Physical activity and body fat percentage in females is statistically non-significant and they have a direct correlation which means that as physical activity increases, body fat percentage also increases. This could be because majority of subjects taken for this study have normal BMI and moderate levels of physical activity. Body fat percentage may decrease with increasing physical activity because of excessive calories expenditure with increasing bodily activities, better fat metabolism, etc.

Graph number 10 shows that Correlation between Physical activity and Body Density for both males and females is non-significant $(\mathrm{p}=0.713)$ in males and $(\mathrm{p}=0.618)$ in females but there exists a direct correlation (Pearson correlation $=0.083$ ) between them in males and inverse correlation (Pearson correlation=0.067) between them in females. Body density is how much space you take up for every square inch you weigh, essentially your compactness. ${ }^{[12]}$ Direct correlation between them in males suggests that as physical activity increases, body density also increases and inverse correlation between them suggests that as physical activity increases, body density decreases. The non-significance could be because of smaller sample size and unequal distribution of males and females and also because majority of the subjects have normal BMI and majority have moderate levels of physical activity.

Graph number 11 shows that Correlation between Physical activity and Age for both males and females is nonsignificant $(\mathrm{p}=0.402$ in males and $\mathrm{p}=0.150$ in females but there exists a direct correlation (Pearson correlation $=0.188$ in males and Pearson correlation $=0.191$ in females) between them. Direct correlation between Physical activity and age in males and females suggests that physical activity increases with age. As responsibilities increases with increasing age, so does the amount of duties like earning for family, cooking, marketing, doing various household chores like sweeping, dusting, brooming, etc. traveling to and fro places; which may lead to increase in Physical activity. Hence, it can be justified that as Physical activity increases with increasing age.

A study carried out by Int J Yoga suggested that physical activity alone cannot maintain BMI and body fat percent, but it can reduce the risk of overweight and high body fat percent in the population. ${ }^{[13]}$ Individuals who are overweight and obese may have decreased levels of physical activity and greater waist circumference and body fat percentage. Thus, importance of Physical activity, its advantages, correlation with anthropometric measures should be studied and efforts should be taken towards healthy lifestyle.

\section{Conclusion:-}

1. Physical activity v/s BMI- correlation between Physical activity and Body mass index (BMI) for both males and females is statistically non-significant but there exists a direct correlation between them.

2. Physical Activity v/s Waist circumference - correlation between Physical activity and waist circumference for both males and females is statistically non-significant but there exists a direct correlation between them.

3. Physical activity v/s Body fat percentage- correlation between Physical activity and Body fat percentage for both males and females is statistically non-significant but there exists an inverse correlation between Physical activity and body fat percentage in males and direct correlation between physical activity and body fat percentage in females.

4. Physical activity v/s Body Density -Correlation between Physical activity and Body Density for both males and females is statistically non-significant but there exists a direct correlation between Physical activity and body density in males and inverse correlation between Physical activity and Body density in females.

5. Physical activity v/s Age -correlation between Physical activity and Age for both males and females is statistically non-significant but there exists a direct correlation between them.

\section{Limitations:-}

1. Small sample size

2. Non uniform distribution of population as Females were more than males.

3. Improper distribution of BMI as majority samples belonged to normal body mass index.

\section{Recommendations:- For Young Adults:}


Awareness about importance of Physical activity- Aerobic exercise is very important and has many advantages. It is an important aspect of healthy lifestyle. It includes various activities such as jogging, swimming, cycling, walking, etc. Risk of various diseases such as hypertension, blood pressure, etc. can be lowered with regular aerobic exercise. Exercises maybe done following FITT principle.

1. Frequency is how often you exercise i.e. number of days per week.

2. Intensity is how hard your exercise. This could be low, moderate, or high intensity.

3. Time refers to the time of day you exercise and for how long.

4. Type refers to what kind of exercise you are doing like strength training, circuit training, etc.

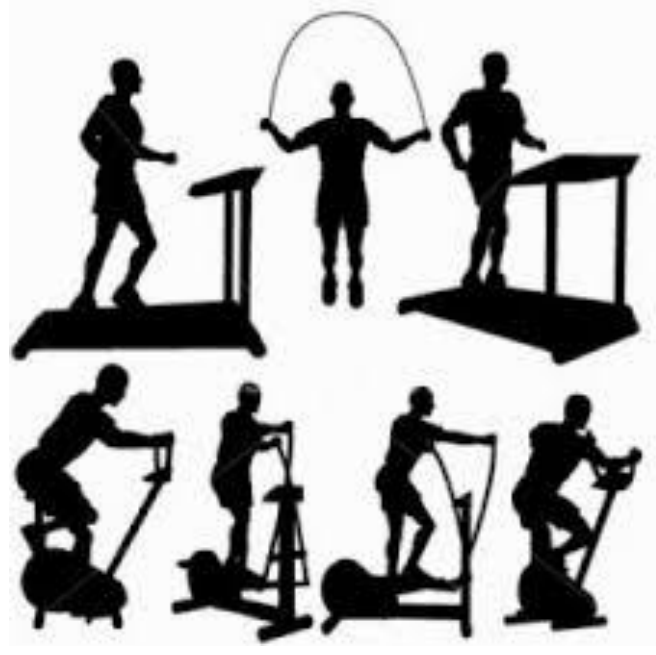

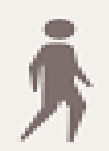

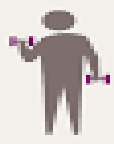

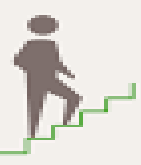

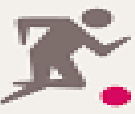

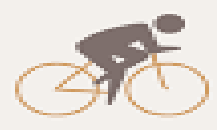

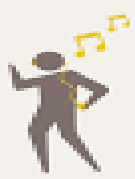

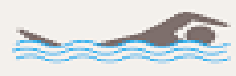

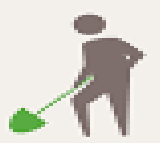

1. Awareness to maintain ideal body compositions- Encouragement for healthy lifestyle and suggesting modifications like regular walk, regular exercises, use stairs instead of elevators, carrying our chores in standing rather than sitting can be suggested.

2. Dietary modifications can be advised like less consumption of carbohydrates and fats (triglycerides), decrease intake of salts, sweets. Restrict intake of oil, cheese, white rice bread.

3. Encourage the young adults to have more of fruits, salads and high fiber diet. 


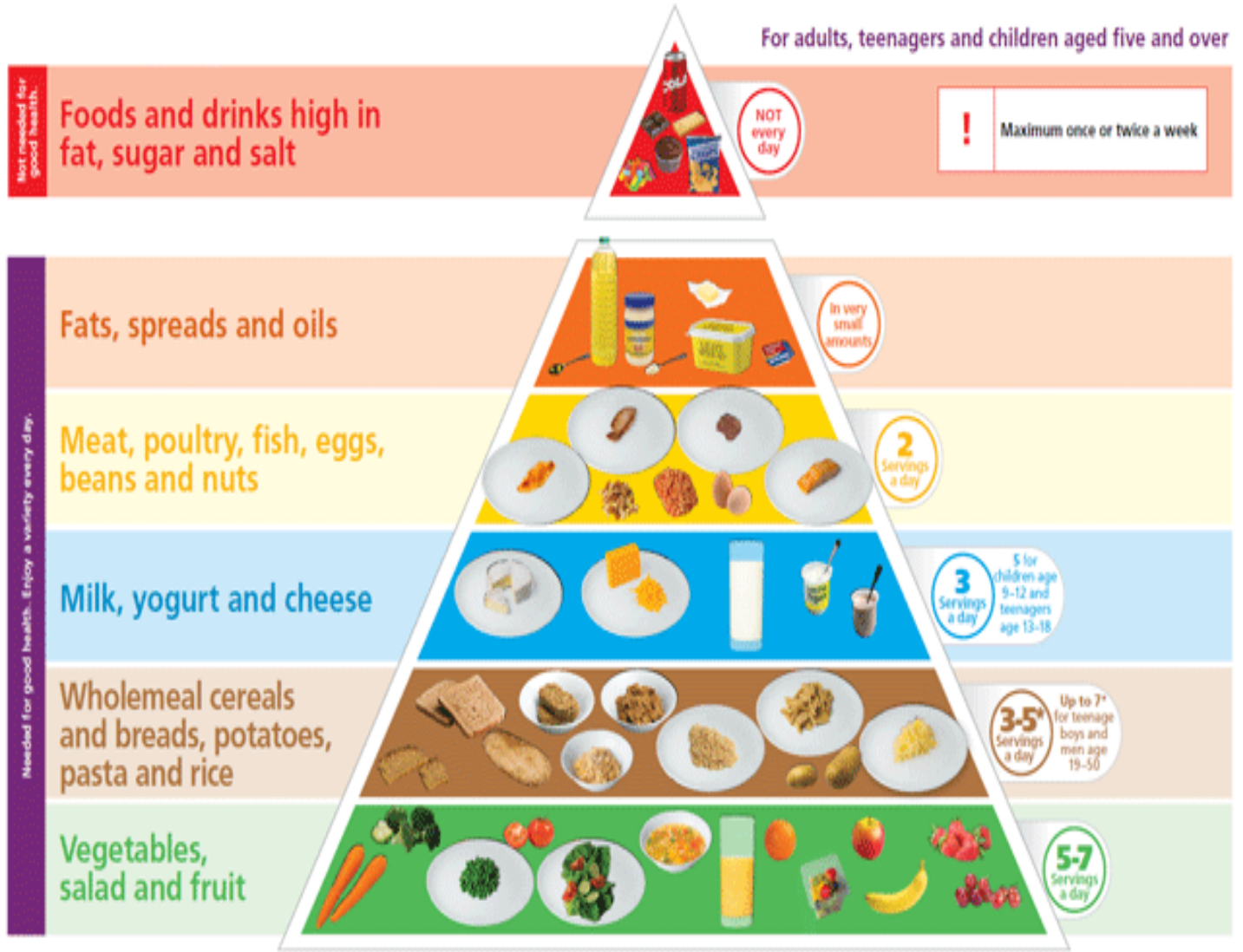

4. To spread awareness about risks of obesity and physical inactivity

\section{For further research:-}

1. Larger sample size can be taken

2. Homogeneous distribution of samples in all categories of BMI and gender as well should be considered.

3. Similar study can be further extended in different age groups.

\section{Acknowledgement:-}

1. I take this opportunity to express my sincere gratitude to those people whose support and concern this project would not have been a success.

2. I am also extremely thankful to Dr. Unnati Pandit, Director and Professor, who has given me the permission to initiate the project in this institution and has given her valuable advice and help throughout.

3. I am extremely thankful to my guide Dr. Sukhada Prabhu, for her valuable guidance, for giving her valuable time and help throughout.

4. And last but not the least, the colleagues of our branch and my study subjects who deserve a word of thanks for their co-operation. 


\section{References:-}

1. Physical activity [Internet]. World Health Organization. World Health Organization; 2018 [cited 2019Feb10].

2. Ahmad N, Adam SI, Nawi AM, Hassan MR, Ghazi HF. Abdominal obesity indicators: Waist circumference or waist-to-hip ratio in Malaysian adults population. International journal of preventive medicine. 2016;7.

3. Stalker GL. Reliability and Validity of the Body Caliper to Evaluate Body Composition.

4. Baranauskas MN, Johnson KE, Juvancic-Heltzel JA, Kappler RM, Richardson L, Jamieson S, Otterstetter R. Seven-site versus three-site method of body composition using BodyMetrix ultrasound compared to dual-energy X-ray absorptiometry. Clinical physiology and functional imaging. 2017 May;37(3):317-21.

5. Obesity [Internet]. World Health Organization. World Health Organization; 2014 [cited 2019Feb10].

6. Ng M, Fleming T, Robinson M, Thomson B, Graetz N, Margono C, Mullany EC, Biryukov S, Abbafati C, Abera SF, Abraham JP. Global, regional, and national prevalence of overweight and obesity in children and adults during 1980-2013: a systematic analysis for the Global Burden of Disease Study 2013. The lancet. 2014 Aug 30;384(9945):766-81.

7. Meharda B, Sharma SK, Singhal G, Kumar D. Overweight and obesity: a rising problem in India. International Journal Of Community Medicine And Public Health. 2017 Nov 23;4(12):4548-52.

8. Undavalli VK, Ponnaganti SC, Narni H. Prevalence of generalized and abdominal obesity: India's big problem. International Journal Of Community Medicine And Public Health. 2018 Mar 23;5(4):1311-6.

9. World Health Organization. Waist circumference and waist-hip ratio: report of a WHO expert consultation, Geneva, 8-11 December 2008.

10. Stalker GL. Reliability and Validity of the Body Caliper to Evaluate Body Composition.

11. Jaafari L. Health-related anthropometric measures in connection with physical fitness factors. International Proceedings of Economics Development and Research. 2012;31:21.

12. Andrea. Definition of Body Density [Internet]. Livestrong.com. Leaf Group; [cited 2019Feb12].

13. Kesavachandran C, Bihari V, Mathur N. Can physical activity maintain normal grades of body mass index and body fat percentage?. International journal of yoga. 2009 Jan;2(1):26. 